Article

\title{
Burst Misalignment Evaluation for ALOS-2 PALSAR-2 ScanSAR-ScanSAR Interferometry
}

\author{
Ryo Natsuaki ${ }^{1}{ }^{*}$, Takeshi Motohka ${ }^{1}$, Masanobu Shimada ${ }^{2}$ and Shinichi Suzuki ${ }^{1}$ \\ 1 Earth Observation Research Center, Japan Aerospace Exploration Agency, 2-1-1 Sengen, Tsukuba 305-8505, \\ Ibaraki- Prefecture, Japan; motooka.takeshi@jaxa.jp (T.M.); suzuki.shinichi@jaxa.jp (S.S.) \\ 2 School of Science and Engineering, Tokyo Denki University, Ishizaka, Hatoyama-machi, Hiki-gun 350-0394, \\ Saitama Prefecture, Japan; shimada@g.dendai.ac.jp \\ * Correspondence: natsuaki.ryo@jaxa.jp; Tel.: +81-50-3362-7567
}

Academic Editors: Bruce Chapman, Paul Siqueira, Nicolas Baghdadi and Prasad S. Thenkabail Received: 21 December 2016; Accepted: 25 February 2017; Published: 28 February 2017

\begin{abstract}
This paper reports the validation results of burst misalignment for ScanSAR-ScanSAR interferometry of the Phased Array-type L-band Synthetic Aperture Radar-2 (PALSAR-2) aboard the Advanced Land Observing Satellite-2 (ALOS-2, "DAICHI-2"). After the internal software modification on 8 February 2015, ALOS-2 ScanSAR observation mode archives have been available for use in interferometric analysis, as it was planned. However, it has not been reported whether its burst misalignment satisfies the mission requirements: $90 \%$ or higher burst overlap ratio. The validation results in this paper confirm that the expected observation misalignment satisfies the required range, including minimal seasonal and orbital dependencies. The results in this paper are obtained directly from the azimuth offset of the single look complex (SLC) data and are not derived from the orbit records. Nine identical orbits and frame numbers are chosen and evaluated to investigate the average burst misalignment and orbital dependency.
\end{abstract}

Keywords: synthetic aperture radar (SAR); ALOS-2; PALSAR-2; ScanSAR; ScanSAR-ScanSAR interferometry

\section{Introduction}

Synthetic Aperture Radar (SAR) has become an essential tool for the rapid analysis of, for example, disaster monitoring [1], maritime monitoring and land cover changes [2,3] including environmental monitoring [4]. Especially for a large-scale deformation, interferometric analysis (InSAR) in a wide-swath observation mode is effective for precisely detecting ground displacement [5]. Currently, by using InSAR analysis, most SAR satellites support centimeter accuracy from dozens of kilometers of observation swaths when detecting displacements. The spatial resolution of SAR depends on its bandwidth and observation mode, thus it varies from sub-meters to hundreds of meters. For wider swath requirements, SAR satellites provide a specific low resolution and wide swath observation mode. In this so-called ScanSAR mode, a SAR satellite cyclically switches the antenna look angle to different slant range positions during observation. The focused data have hundreds of kilometers of swath with relatively worse resolution compared to ordinal Stripmap SAR images [6]. In the case of the Terrain Observation by Progressive Scans (TOPSAR) mode, SAR also sweeps the antenna look angle toward the along-track direction [7]. These wide swath modes support InSAR analysis only if their switching timing is aligned between two observations [8,9]. If the timing is not aligned, the interferogram contains poor or no interferometric coherence. A wide swath interferogram is important when investigating a large-scale ground deformation such as the April 2015 Nepal earthquake (Mw 7.8 Gorkha, Nepal earthquake) and its aftershocks. In this case, the $350 \mathrm{~km}$ swath Advanced Land 
Observing Satellite-2 (ALOS-2, "DAICHI-2") ScanSAR and the $250 \mathrm{~km}$ swath Sentinel-1A TOPSAR covered the $170 \mathrm{~km}$ wide deformation area.

The Phased Array-type L-band Synthetic Aperture Radar-2 (PALSAR-2) aboard ALOS-2 is the latest L-band spaceborne SAR operated by the Japan Aerospace Exploration Agency (JAXA) [10]. ALOS-2 has a worldwide observation scenario for various missions, e.g., deformation detection, forest change monitoring and sea ice monitoring. Its precise orbit control and high-power radar enable accurate analysis with high spatial resolution. ALOS-2 mainly observes the world with a $10 \mathrm{~m}$ resolution/70 km swath Stripmap mode and a $100 \mathrm{~m}$ resolution/350 km swath ScanSAR mode. The $3 \mathrm{~m}$ resolution Stripmap mode is mainly used for the Japan region while Spotlight mode is used for some specific places. ALOS-2 has a frequent 14-day revisit cycle, which is three times faster than the previous ALOS (46-day cycle). Because of the limitations in swath width and data downlinking, the scheduled cycle in the basic observation scenario is longer than 14 days. However, if a large disaster occurs, ALOS- 2 will be rescheduled to observe the affected area for a specific duration during every revisit cycle. Using these features, ALOS-2 performed more than 130 emergency observations in 2015 [11].

ALOS-2 ScanSAR mode is programed to support InSAR analysis by achieving an along-track positional accuracy of less than $10 \mathrm{~m}$. In this case, ALOS-2 ScanSAR images have a burst overlap ratio that will be larger than $90 \%$ in 1 sigma, i.e., a pair of ScanSAR images has a burst overlap ratio of more than $90 \%$ with a probability of $68.3 \%[12,13]$.

ALOS-2 system records confirm that after the system modification on 8 February 2015, the burst misalignment satisfies the above requirements [14]. The misalignment pattern before 8 February 2015 has been investigated in $[15,16]$ by comparing against the data acquired after the modification. However, the precise overlap ratio/misalignment after the modification have not been evaluated to date. Its time and orbit dependencies have not been investigated. In other words, the modification has been evaluated from the system record and not from the acquired data, though the system was once implemented incorrectly. Actually, some reports have noted that a minor time dependency may exist $[17,18]$ though its precise frequency has not been investigated. If there is any remaining time dependency and if it can be subtracted, the burst overlap ratio can be improved. This paper presents these results and confirms that the offset ratio of the images after 8 February are aligned better than planned. The frequency of the time dependency is also investigated.

\section{ScanSAR InSAR and Burst Misalignment Detection}

In this section, the burst misalignment of ScanSAR InSAR is briefly explained. Next, the misalignment evaluation method applied in this paper is described. Some terms and parameters are specified for the ALOS-2 ScanSAR mode.

\subsection{ALOS-2 ScanSAR Mode}

In ScanSAR observation mode, ALOS-2 cyclically switches the observation angle to five or seven neighboring different slant range positions (subswaths) and achieves a $350 \mathrm{~km}$ or $490 \mathrm{~km}$ total observation swath, respectively. In the worldwide observation scenario, a five subswath $350 \mathrm{~km}$ width mode is used for land observation while a seven subswath $490 \mathrm{~km}$ width mode is used for maritime observation. The planned requirement of the ALOS-2 ScanSAR mode is that the burst overlap ratio should be larger than $90 \%$ in 1 sigma for the five subswath mode for the interferometric analysis. This is equal to a burst misalignment better than $15.8 \mathrm{~ms}$. The definition of burst overlap ratio and burst misalignment are as follows:

In the case of the five subswath mode, ALOS-2 transmits a radar pulse from subswath number 1 (nearest) to number 5 (farthest). Transmission of the radar pulse to one subswath is known as a "burst," and the burst duration for each subswath is equal to one-fifth of the total switching cycle. The cycle includes the delay time for switching the beam angle from one subswath to another. The burst overlap ratio $\mathrm{BOR}$ is: 


$$
\mathrm{BOR}=\frac{B_{0}}{B_{d u r}}
$$

where $B_{d u r r}$ is the burst duration and $B_{o}$ is the length of the burst overlap between the master and slave. Burst misalignment $B_{\text {mis }}$ is

$$
B_{\text {mis }}=t_{\text {est }}-t_{\text {true }}
$$

where $t_{\text {est }}$ and $t_{\text {true }}$ are the actual and ideal starting times of the burst, respectively. If there is an interferometric pair, $B_{\text {mis }}$ becomes the misalignment of their burst cycle and

$$
B_{o}=B_{d u r}-B_{m i s}
$$

In summary, the burst overlap ratio presents a signal ratio that can be used for interferometric analysis while the burst misalignment represents the accuracy of the SAR system. In ALOS-2, one cycle of switching is fixed to approximately $0.79 \mathrm{~s}, B_{d u r}=0.158 \mathrm{~s}$ for each subswath, and the required $\mathrm{BOR}=0.9$; thus, the required $B_{\text {mis }}=1.58 \times 10^{-2} \mathrm{~s}$. Note that ALOS-2 adjusts $B_{d u r}$ of the subswath 5 temporarily so that it can start transmitting the pulse in subswath 1 at ideal timing [19]. This adjustment is small (e.g., 10 out of 500 pulses) and ignored in this paper.

The requirement of the burst overlap ratio was however not fulfilled until 8 February 2015, as the parameter for estimating the satellite latitude (along-track position) was set incorrectly. This problem led to the burst overlap ratio being dependent on the acquisition dates of the two ScanSAR images. It was also found that this problem affected the burst overlap ratio but did not affect the geometrical accuracy. JAXA announced in [14] that the along-track shift can be calculated from its acquisition date. This equation is derived from the orbital record of ALOS-2. They re-calculated the difference between the actual and incorrectly set burst alignment positions. Lindsey et al. [15] independently analyzed the burst overlap ratio from the archive of ALOS-2 single look complex (SLC) data and found that the sinusoidal oscillation was approximately the same as the official one. They counted the actual offset of two SLC images by cross-correlation co-registration and fitted it to a sine function. These two functions led to almost the same offsets, i.e., either of the functions can be used for offset prediction of the ScanSAR images acquired prior to 8 February 2015 [16].

After the modification, ALOS-2 ScanSAR images have been able to support InSAR analysis. However, the burst overlap ratio after modification has not been evaluated to date.

\subsection{Burst Misalignment Detection}

To calculate the burst overlap ratio of ALOS-2, the ideal observation timing must be estimated. Because there is no ideal burst timing data, this needs to be estimated from the observation records. If the observation timing contains randomness, the distribution of the burst misalignment between two observations will be random too. If the timing contains seasonal dependency, the distribution will contain a time component. If the ideal and actual observation timings are known, this estimation process is unnecessary. However, ALOS-2 does not provide the ideal timing. Therefore, estimation of the ideal timing $B_{\text {mis }}=0$ should be performed prior to calculating the misalignment.

In this paper, the $B_{\text {mis }}=0$ is estimated from azimuth co-registration between the archives, the same as $[15,16]$. The first archive achieved after 8 February 2015, for each orbit is used as the master image for the co-registration. The others are used as the slave images, and they are calculated to estimate the azimuth offset. The average of the offsets represents the relative offset between the first archive, namely, $\Phi_{1}$, and the others. If there are N SLCs and the S th SLC is denoted as $\Phi_{S}$, the offset between $\Phi_{1}$ and $\Phi_{S}$ can be $K\left(\Phi_{1}, \Phi_{S}\right)$, where the bracket $K($, ) denotes a function that estimates the offset from the cross-correlation. The relative offset $\mathrm{D}_{\mathrm{r} 1}$ between $\Phi_{1}$ and the others is

$$
D_{r 1}=\frac{1}{N-1} \sum_{S=2}^{N} K\left(\Phi_{1}, \Phi_{S}\right)
$$


If there is no systematic timing error and the number of the archive is large enough, $D_{r 1}$ is equal to the absolute offset between the ideal timing and $\Phi_{1}$, namely, $D_{a 1}$. Conversely, the absolute offset of $\Phi_{S}, D_{a S}$, is

$$
D_{a S}=K\left(\Phi_{1}, \Phi_{S}\right)-D_{a 1}
$$

By plotting $D_{a 1}$ to $D_{a N}$, the time dependency of the ScanSAR offset can be estimated. Using multiple orbits/frames, the positional dependency can be estimated using the same operation.

As $D_{a 1}-D_{a N}$ are the results of the cross-correlation maximum, the unit of the offset values is the pixel. Conversely, the azimuth sampling ratio of the SLC depends on the pulse repetition frequency (PRF). ALOS-2 adjusts the PRF automatically and the value of PRF depends on the sub-swaths and satellite position, enabling the burst to be transmitted at the correct timing. Therefore, conversion of the unit from the offset in pixel $\left(D_{a S}\right)$ to the offset in seconds $\left(B_{m i s}\right)$ is required. The conversion equation is

$$
B_{\text {mis }}=\frac{D_{a S}}{P R F}
$$

In this paper, $D_{a S}$ of one sub-swath is calculated from 1000 tie points randomly distributed in the SLC. Then, the $D_{a S}$ of five sub-swaths in one frame are averaged.

\subsection{Doppler Centroid Analysis}

In addition to the error in the burst alignment system, the cross-correlation results include the misalignment derived from the difference of the Doppler centroid frequency $\left(F_{d c}\right)$. The difference of the $F_{d c}$ is generally small because ALOS-2 has a small baseline in both the along- and cross-track direction [19]. The standard product of the ALOS-2 SLC is firstly focused in the native Doppler centroid and then de-skewed toward zero Doppler geometry. If the pointing angle has a difference between two SLCs, the offset derived from the Doppler shift can be observed. When the SLCs have the difference of the $F_{d c}$, namely $d F_{d c}$ and the azimuth FM ratio $K_{a}$, their azimuth offset $D_{F d c}$ is

$$
D_{F d c}=\frac{d F_{d c}}{K_{a}}
$$

In order to estimate the precise $B_{m i s}, D_{F d c}$ is estimated and subtracted in the following sections.

\section{Experimental Results}

In this section, nine identical locations, i.e., nine identical paths/frames were chosen to estimate the azimuth offset of ALOS-2 ScanSAR images. The period of the analysis was 600 days, from 8 February 2015, to 30 September 2016. The path/frame number, flight direction, and number of archives are listed in Table 1. Their positions on the map are shown in Figure 1. All the paths/frames had at least six archives, and the total number of archives was 88 .

Table 1. List of paths and frames used for misalignment estimation.

\begin{tabular}{ccccc}
\hline Name & Path & Frame & Flight Direction & Number of Archives \\
\hline Philippine_P24 & 24 & 3350 & Descending & 8 \\
Philippine_P25 & 25 & 3350 & Descending & 7 \\
Indonesia_P29 & 29 & 3650 & Descending & 11 \\
Antarctica_P53 & 53 & 5050 & Descending & 7 \\
Gabon_P88 & 88 & 3650 & Descending & 14 \\
Croatia_P92 & 92 & 2700 & Descending & 13 \\
Hokkaido_P123 & 123 & 850 & Ascending & 6 \\
Napa_P168 & 168 & 2850 & Descending & 12 \\
Napa_P169 & 169 & 2850 & Descending & 10 \\
\hline
\end{tabular}




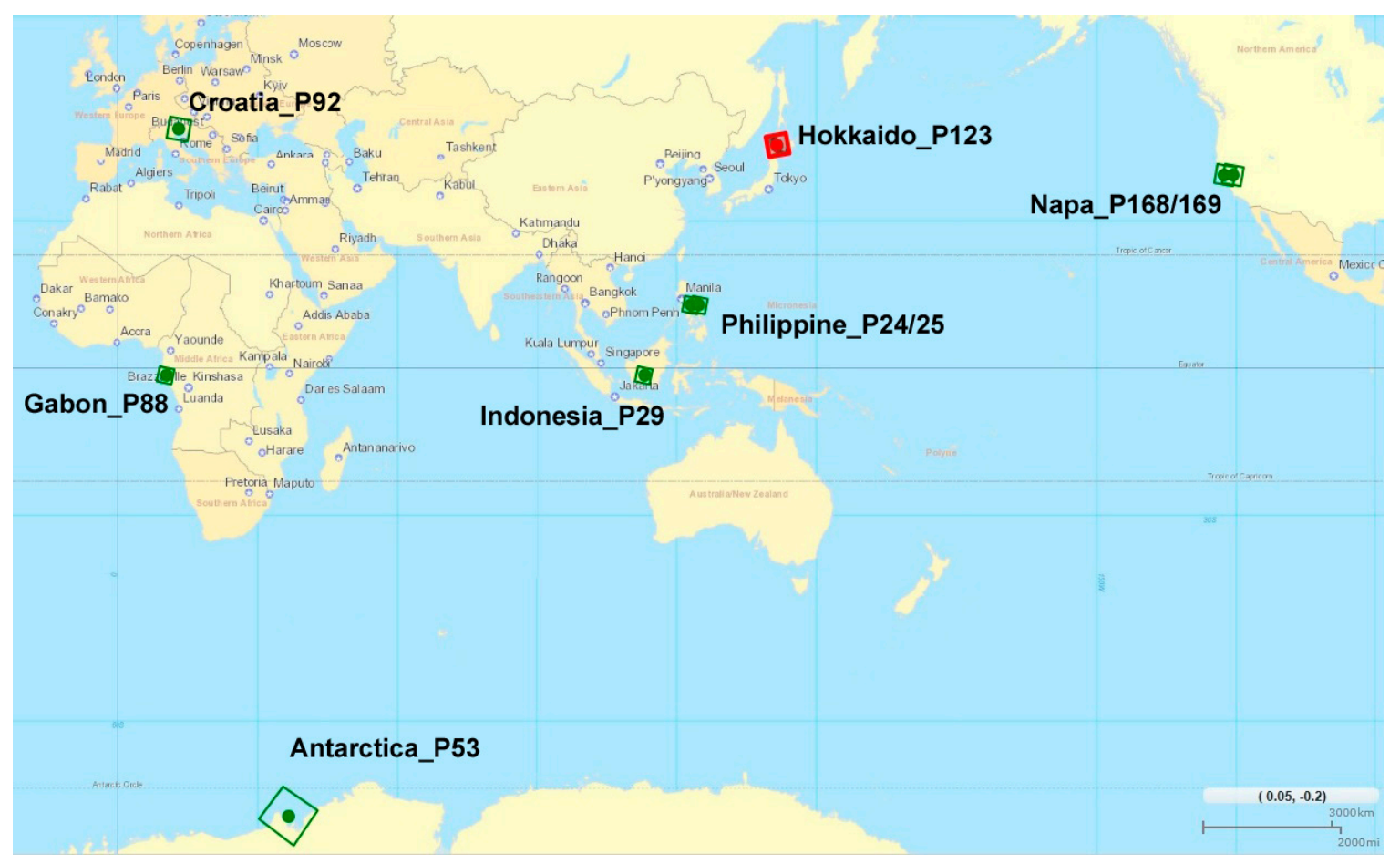

Figure 1. Positions of the sampled paths/frames.

ALOS-2 has a worldwide observation scenario and the ScanSAR mode is mainly used in descending mode (the flight direction from north to south at noon). Therefore, all paths except Hokkaido_P123 are descending. By using the Philippine and Napa archives, the difference between the neighboring paths is compared. According to the ALOS-2 basic observation scenario, a maximum of fifteen acquisitions are expected for the worldwide descending ScanSAR observation, while seven acquisitions are expected for the Japan ascending ScanSAR observation from 8 February 2015, to 30 September 2016. However, due to the observation strategy, polar regions e.g., Antarctica_P53, have less scheduled observations on purpose. In addition, some acquisitions were cancelled due to conflict with emergency observations in response to natural hazards such as earthquakes, storms and floods. Note that all data and parameters are derived from the ALOS-2 standard products.

Figure 2 shows the misalignment plot caused by the Doppler shift. Two large shifts, Philippine_P24 at 11 April 2015 (9.49 ms) and Napa_P168 at 16 October 2015 (13.02 ms), were found. However, the standard deviation (STD) of the Doppler shifts was $1.93 \mathrm{~ms}$ including the large shifts. The large shifts were probably caused by the orbital maneuver soon before the observation. In other words, if the burst alignment system had no error nor randomness, the misalignment of the ALOS-2 ScanSAR data will be 1.93 ms caused by the Doppler centroid shift and has $99 \%$ in the burst overlap ratio.

Figure 3 presents a histogram of the remaining misalignment $B_{\text {mis }}$ after subtracting the Doppler shift in Figure 2. If the misalignment becomes large $(>0)$, ALOS-2 is observed after its ideal timing. That is, the actual observation timing is later than the ideal timing. If the misalignment is smaller than zero $(<0)$, ALOS-2 is observed before the ideal timing. Note that the "ideal" orbit in this article is estimated from the archives as described in the previous section. The STD was $11.37 \mathrm{~ms}$, smaller than the designed misalignment. That is, the misalignment of the ALOS-2 ScanSAR data is better than $11.37 \mathrm{~ms}$ and has $93 \%$ in the overlap ratio with a probability of $68.3 \%$. 


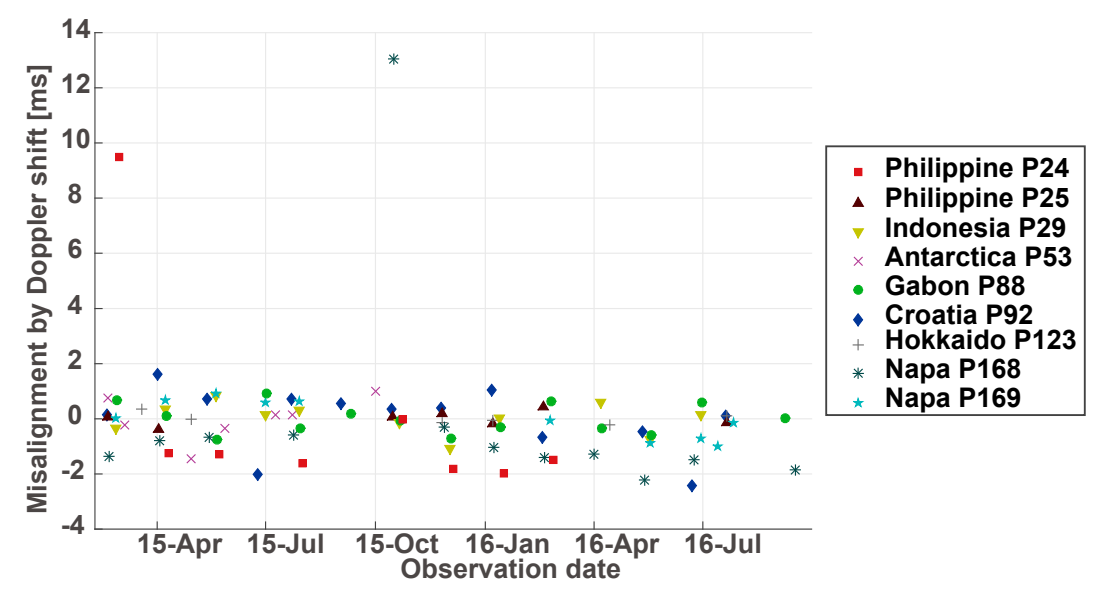

Figure 2. Misalignment caused by the Doppler shift.

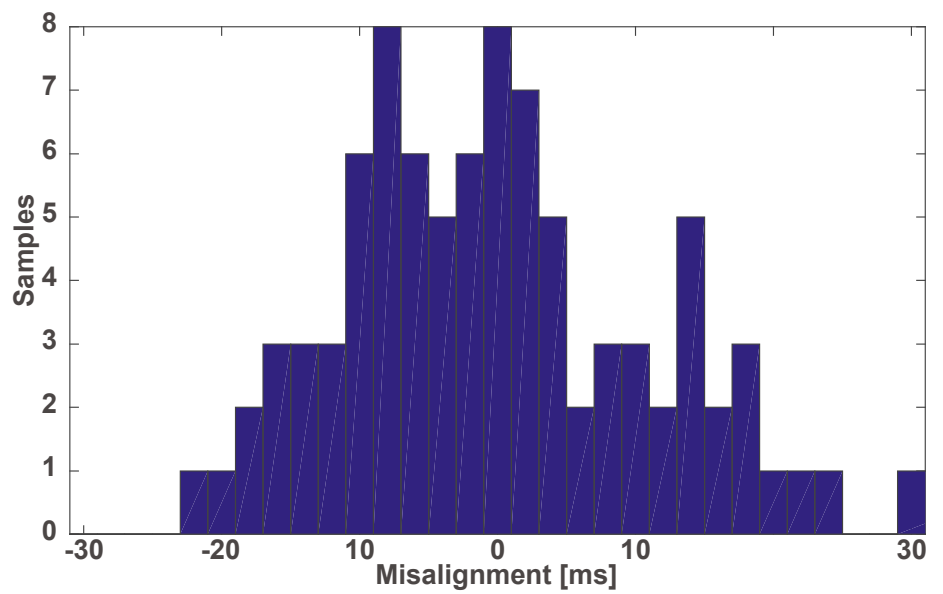

Figure 3. Remaining misalignment from ideal timing after subtracting the Doppler shift.

Figure 4 presents the misalignment plot for nine paths. Some paths, for example, Gabon_P88, Napa_P168 and Napa_P169 have a larger time dependency than others. However, all their peaks are aligned, and these observed misalignments strongly suggest that there is a remaining time dependency in the autonomous burst alignment system in ALOS-2. Note that from the estimated overall accuracy, the burst overlap ratio including the time dependency and Doppler centroid shift fulfills the requirements. Table 2 shows the latest, earliest and STD of the misalignment for each path. From the two close-path pairs, Philippine_P24 and P25, and Napa_P168 and P169, no dependency was found for the latitude or longitude of the path and frame. Napa_P168 and Gabon_P88 tend to mark later and earlier observations compared with the others. In particular, in Gabon_P88, the worst misaligned pair with a $59.01 \mathrm{~ms}$ or a $63 \%$ burst overlap ratio was marked. Note that a $63 \%$ burst overlap ratio is better than the minimum requirement of $30 \%$ and high enough to perform interferometric analysis. Conversely, from the results of Hokkaido_P123, the orbit direction does not affect the misalignment. Moreover, neither does bandwidth affect the misalignment because the Japan area including Hokkaido_P123 is observed with $28 \mathrm{MHz}$ bandwidth while the other paths are observed with $14 \mathrm{MHz}$. The solid line in Figure 3 shows the approximated sinusoidal curve function derived from a full search. It was

$$
B_{\text {mis }}(t)=-1.7\left(\frac{t}{100}\right)+6+11 \sin \left(2 \pi\left(\frac{137+t}{347}\right)\right)
$$

where $t$ is the date after 8 February 2015. The coefficient of determination (adjusted $R^{2}$ ) of (8) was 0.54 . 


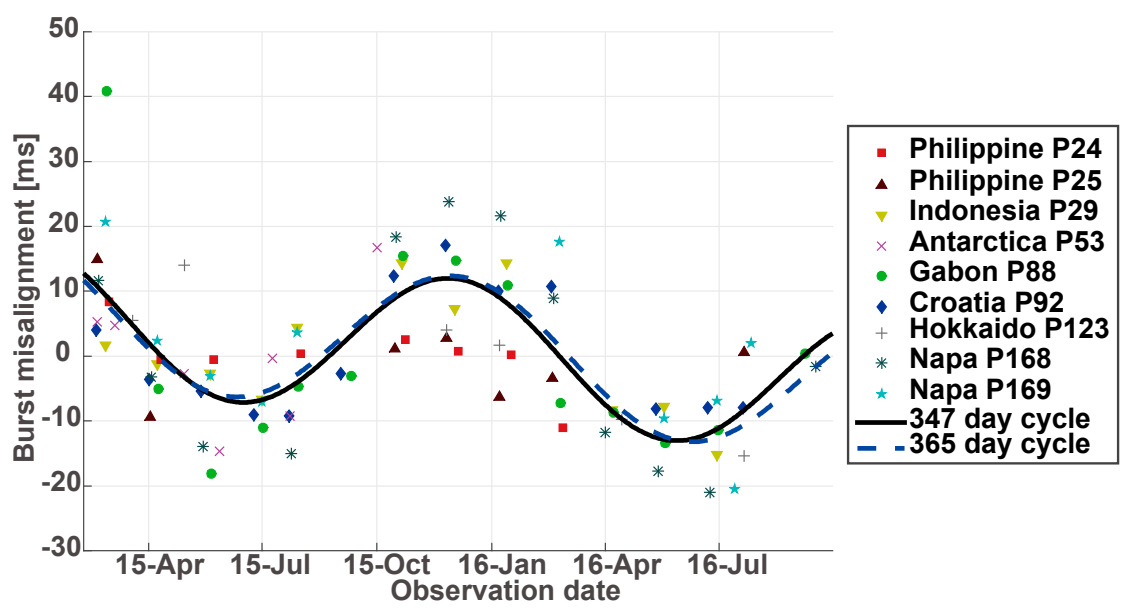

Figure 4. Time dependency of the misalignment of each path.

Table 2. Misalignment record of each path in milliseconds. The average misalignment is set to zero.

\begin{tabular}{ccccc}
\hline Name & $\begin{array}{c}\text { Latest } \\
\text { Misalignment }\end{array}$ & $\begin{array}{c}\text { Earliest } \\
\text { Misalignment }\end{array}$ & $\begin{array}{c}\text { Standard } \\
\text { Deviation }\end{array}$ & $\begin{array}{c}\text { Misalignment } \\
\text { of Worst Pair }\end{array}$ \\
\hline Philippine_P24 & 8.38 & -11.13 & 5.37 & 19.51 \\
Philippine_P25 & 14.81 & -9.46 & 7.86 & 24.27 \\
Indonesia_P29 & 14.44 & -15.19 & 9.50 & 29.64 \\
Antarctica_P53 & 16.74 & -14.62 & 10.30 & 31.36 \\
Gabon_P88 & 40.90 & -18.10 & 15.67 & 59.01 \\
Croatia_P92 & 17.11 & -9.20 & 9.50 & 26.31 \\
Hokkaido_P123 & 14.03 & -15.34 & 10.74 & 29.37 \\
Napa_P168 & 23.70 & -21.03 & 16.27 & 44.73 \\
Napa_P169 & 20.77 & -20.40 & 12.37 & 41.16 \\
\hline
\end{tabular}

\section{Discussion}

In this section, the origin of time dependency is discussed. As shown in the previous section, the burst misalignment of ALOS-2 depends on the acquisition date. If this is simply derived from a small number of samples, the time dependency will be random for each orbit. However, the plots align with each other, as shown in Figure 4. From their seasonal patterns, it can be assumed that some components of the precession and/or nutation of the Earth are not included in the orbit prediction system of ALOS-2. Actually, the system includes their main components; however, some minor components may be omitted. In such a case, the approximated curve will be 365-day cycle sinusoidal. To enable the further analysis, the 365-day cycle curve was approximated as Equation (9) from a full search.

$$
B_{\text {mis }}(t)=-1.9\left(\frac{t}{100}\right)+7+11 \sin \left(2 \pi\left(\frac{157+t}{365}\right)\right)
$$

where $t$ is the date after 8 February 2015. The coefficient of determination (adjusted $R^{2}$ ) of Equation (9) was 0.52 . The curve is plotted as a dashed line in Figure 4.

The extreme values for Equations (8) and (9) were calculated as shown in Table 3. As a result, the extreme values mostly distribute within two weeks. That is, the first local minimum (earliest observation) distributes within 5 days of June in 2015, while the local maximums (latest observation) were on 27 and 28 November 2015. As their adjusted $R^{2}$ were 0.54 and 0.52 , the difference of two functions is small. The root mean squared errors (RMSEs) of these curves are $7.66 \mathrm{~ms}$ and $7.65 \mathrm{~ms}$. That is, if this seasonal component was subtracted by these equations, the burst misalignment will shrink to $7.66 \mathrm{~ms}$ and $7.65 \mathrm{~ms}$ for a 347-day cycle and a 365-day cycle, respectively. Here again, 
two curves are close to each other. The derived 347-day cycle is probably over fitted to the observation results. In addition, there is no 347-day cycle in the Earth's precession and nutation.

The conclusion of this section is that though the exact equation and the origin were not derived in this paper, the remaining cycle of the ALOS-2 ScanSAR misalignment may be approximated to one year. The parameter in the autonomous burst alignment system needs to be revised if further accurate burst alignment is required. If the proposed Equation (9) is correct, the burst misalignment will shrink from $11.37 \mathrm{~ms}$ to $7.65 \mathrm{~ms}$ in STD.

Table 3. Extreme values and the days between the first and the third extreme values (peak-to-peak).

\begin{tabular}{ccccc}
\hline \multirow{2}{*}{ Name } & \multicolumn{3}{c}{ Extreme Values } & \multirow{2}{*}{ RMSE (ms) } \\
\cline { 2 - 4 } & First & Second & Third & \\
\hline 347-day cycle (8) & 16 June 2015 & 27 November 2015 & 28 May 2016 & 7.66 \\
365-day cycle (9) & 11 June 2015 & 28 November 2015 & 10 June 2016 & 7.65 \\
\hline
\end{tabular}

\section{Conclusions}

In this paper, validation of ScanSAR burst overlapping for ALOS-2 PALSAR-2 is reported. The burst overlap ratio is confirmed to be better than the planned accuracy, which was a $90 \%$ overlap ratio or $15.8 \mathrm{~ms}$ misalignment with a probability of $68.3 \%$. The actual value was a $93 \%$ overlap ratio or $11.37 \mathrm{~ms}$ misalignment. Orbit direction (ascending/descending) and bandwidth did not affect the misalignment. The misalignment caused by the Doppler shift is also calculated and expected to be $1.93 \mathrm{~ms}$ in STD. However, the remaining burst misalignment still showed a minor seasonal dependency. The frequency of the remaining component was estimated from the approximated sinusoidal function. The derived frequency of the best fitted curve was 347 days. If this remaining component was subtracted, the burst misalignment will shrink to $7.66 \mathrm{~ms}$. Analytical results suggested that the frequency may be 365 days and the 347-day cycle can be the result of over fitting. The standard deviation of the misalignment varied from orbit to orbit. Some orbits achieved a better burst overlap ratio than others. The reason is an open question, though the experimental results strongly suggest that the remaining misalignment was not random but systematic. Note that the current misalignment including the Doppler centroid shift is negligible for interferometric analysis and the burst overlap ratio fulfills the requirements.

Acknowledgments: The authors thank ALOS-2 Calibration and Validation teams for their contribution to the ALOS-2 mission.

Author Contributions: R.N. and M.S. conceived and designed the experiments; R.N. performed and analyzed the experiments; T.M. evaluated experimental results with observation records; M.S. and S.S. managed this research.

Conflicts of Interest: The authors declare no conflict of interest.

\section{References}

1. Matsuoka, M.; Nojima, N. Building Damage Estimation by Integration of Seismic Intensity Information and Satellite L-band SAR Imagery. Remote Sens. 2010, 2, 2111-2126. [CrossRef]

2. Chapman, B.; McDonald, K.; Shimada, M.; Rosenqvist, A.; Schroeder, R.; Hess, L. Mapping Regional Inundation with Spaceborne L-Band SAR. Remote Sens. 2015, 7, 5440-5470. [CrossRef]

3. Brown, D.R.N.; Jorgenson, M.T.; Kielland, K.; Verbyla, D.L.; Prakash, A.; Koch, J.C. Landscape Effects of Wildfire on Permafrost Distribution in Interior Alaska Derived from Remote Sensing. Remote Sens. 2016, 8. [CrossRef]

4. Shimada, M.; Itoh, T.; Motooka, T.; Watanabe, M.; Shiraishi, T.; Thapa, R.; Lucas, R. New global forest/non-forest maps from ALOS PALSAR data (2007-2010). Remote Sens. Environ. 2014, 155, $13-31$. [CrossRef]

5. Wen, Y.; Xu, C.; Liu, Y.; Jiang, G. Deformation and Source Parameters of the 2015 Mw 6.5 Earthquake in Pishan, Western China, from Sentinel-1A and ALOS-2 Data. Remote Sens. 2016, 8. [CrossRef] 
6. Moore, R.K.; Claassen, J.P.; Lin, Y.H. Scanning Spaceborne Synthetic Aperture Radar with Integrated Radiometer. IEEE Trans. Aerosp. Electron. Syst. 1981, AES-17, 410-421. [CrossRef]

7. De Zan, F.; Guarnieri, A.M. TOPSAR: Terrain Observation by Progressive Scans. IEEE Trans. Geosci. Remote Sens. 2006, 44, 2352-2360. [CrossRef]

8. Bamler, R.; Eineder, M. ScanSAR processing using standard high precision SAR algorithms. IEEE Trans. Geosci. Remote Sens. 1996, 34, 212-218. [CrossRef]

9. Plank, S. Rapid Damage Assessment by Means of Multi-Temporal SAR-A Comprehensive Review and Outlook to Sentinel-1. Remote Sens. 2014, 6, 4870-4906. [CrossRef]

10. Arikawa, Y.; Saruwatari, H.; Hatooka, Y.; Suzuki, S. PALSAR-2 launch and early orbit operation result. In Proceedings of the IEEE International Geoscience and Remote Sensing Symposium (IGARSS), Quebec, QC, Canada, 13-18 July 2014.

11. Natsuaki, R.; Nagai, H.; Motohka, T.; Ohki, M.; Watanabe, M.; Suzuki, S. Emergency observation and its analysis using ALOS-2 PALSAR-2 in 2015. Proc. SPIE 2016, 9877. [CrossRef]

12. Okada, Y.; Hamasaki, T.; Tsuji, M.; Iwamoto, M.; Hariu, K.; Kankaku, Y.; Suzuki, S.; Osawa, Y. Hardware Performance of L-band SAR System Onboard ALOS-2. In Proceedings of the IEEE International Geoscience and Remote Sensing Symposium (IGARSS), Vancouver, BC, Canada, 24-29 July 2011.

13. Kankaku, Y.; Suzuki, S.; Osawa, Y. ALOS-2 Mission and Development Status. In Proceedings of the IEEE International Geoscience and Remote Sensing Symposium, Melbourne, Australia, 21-26 July 2013.

14. ALOS-2 Project Team. Effective Data for Interferometric Analysis with PALSAR-2 ScanSAR Mode. Available online: https:/ /auig2.jaxa.jp/information_home/ScanSAR_Burst_Overlap_20151127_e.pdf (accessed on 1 December 2016).

15. Lindsey, E.O.; Natsuaki, R.; Xu, X.; Shimada, M.; Hashimoto, M.; Melgar, D.; Sandwell, D.T. Line of sight displacement from ALOS-2 interferometry: M7.8 Gorkha earthquake and Mw 7.3 aftershock. Geophys. Res. Lett. 2015, 42, 6655-6661. [CrossRef]

16. Natsuaki, R.; Nagai, H.; Motohka, T.; Ohki, M.; Watanabe, M.; Thapa, R.B.; Tadono, T.; Shimada, M.; Suzuki, S. SAR interferometry using ALOS-2 PALSAR-2 data for the Mw 7.8 Gorkha Nepal earthquake. Earth Planets Space 2015, 68. [CrossRef]

17. Natsuaki, R.; Motohka, T.; Suzuki, S.; Shimada, M. Burst overlapping of ALOS-2 PALSAR-2 ScanSAR-ScanSAR interferometry. In Proceedings of the CEOS SAR Calibration and Validation Workshop, Tokyo, Japan, 7-9 September 2016.

18. Natsuaki, R.; Motohka, T.; Ohki, M.; Watanabe, M.; Suzuki, S. Validation of burst overlapping for ALOS-2 PALSAR-2 ScanSAR-ScanSAR interferometry. Proc. SPIE 2016. [CrossRef]

19. Shimada, M.; Watanabe, M.; Motohka, T.; Kankaku, Y.; Suzuki, S. Calibration and Validation of the PALSAR-2. In Proceedings of the IEEE International Geoscience and Remote Sensing Symposium (IGARSS), Milan, Italy, 26-31 July 2015.

(C) 2017 by the authors. Licensee MDPI, Basel, Switzerland. This article is an open access article distributed under the terms and conditions of the Creative Commons Attribution (CC BY) license (http:/ / creativecommons.org/licenses/by/4.0/). 